\title{
Souvenirs du monde des montagnes
}

Camille Scherrer

Artist

EPFL+ECAL Lab

11, Av. du 24-Janvier

$\mathrm{CH}$ - 1020 Renens, Switzerland

camille.scherrer@epfl.ch

Julien Pilet

Researcher

Keio University

EPFL/IC/ISIM/CVLab

Station 14

CH-1015 Lausanne, Switzerland

julien@ozawa.ics.keio.ac.jp

Vincent Lepetit

Researcher

CV lab, Ecole Polytechnique

Fédérale de Lausanne

EPFL/IC/ISIM/CVLab

Station 14

$\mathrm{CH}-1015$ Lausanne, Switzerland

vincent.lepetit@epfl.ch

\section{Pascal Fua}

Educator

CV Lab, Ecole Polytechnique

Fédérale de Lausanne

EPFL/IC/ISIM/CVLab

Station 14

CH-1015 Lausanne, Switzerland

pascal.fua@epfl.ch

\section{Camille Scherrer, Julien Pilet, Vincent Lepetit, Pascal Fua}

\begin{abstract}
A B S T R A C T
This paper describes a particular book called Souvenirs du monde des montagnes, which draws its iconography from the history of a Swiss mountain family from 1910 to 1930 . By simply dipping into the first few pages, the reader will be lost between real and virtual universes, wonder about the evolution of the images' meanings, and question an object's true content. This setup, developed using state-of-theart computer vision technology, offers unprecedented freedom: we can make technological references disappear to place the user in fruitful turmoil between visible and hidden meanings. The shadow of a bird flies over the pages, foxes' lanterns light up the text, paper mountains emerge. Once the last page has been turned, the reader will never look at books in the same way again.
\end{abstract}

\section{Introduction}

Our setup, consisting of a book, a lamp, and a computer screen, seems perfectly harmless at first sight. But as soon as you open the book under the lamp, a major confrontation takes place: on the screen, the pages take on new animated and mysterious dimensions. The book's iconography, inspired by a family album of the I930s, changes into a virtual fairy tale. The user is swept away by the disconcerting ambiguity of the setup's language because the virtual animations refer to traditional collages; the viewer hesitates between traditional paper-based media and the virtual

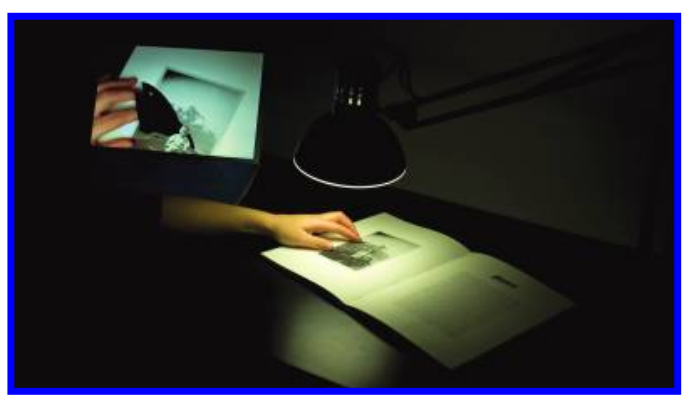

Figure 1. A book and a computer screen showing images taken by a camera hidden in a lamp. This setup offers a new language to bring the real and virtual worlds closer to each other, to weave new meanings between the visible and the invisible. ○ 2009 Camille Scherrer. world. To further increase this confusion, nothing gives away the technology, although in this case it is quite sophisticated. For each of the book's pages, animated worlds miraculously appear and disappear, with a direct connection to the actual items printed on paper: animal figures appear over the mountains, peaks emerge in a shadow play, a bird silently flies over the scene, foxes light up the text with their lanterns at dusk (Figure I). The computer screen acts as a revealer, offering viewers a reality quite different from the reality they perceive when they look directly at an object.
The idea of animating a book is not new: attempts include flip books, motion graphics, and pop-up books [I]. More recently, webcams and computers have made it possible to augment markers printed on the pages of a book with virtual animations [2]. In all these cases the book either disappears or is altered. In our artwork, the book keeps its primary role, and the reader can also enjoy it without the augmentations.

The installation's secret is based on a camera hidden in the lamp, but also on advanced augmented-reality software. The integration of technology is successful for two reasons. First, no technical element distracts users or makes usage difficult, as opposed to augmented-reality 
works that require assistance or visually invasive markers [3,4]. Using a simple camera, our software is able to use the book illustrations themselves as "guides" to integrate our virtual elements. Second, our animations enhance the real book without superseding it. Both electronic

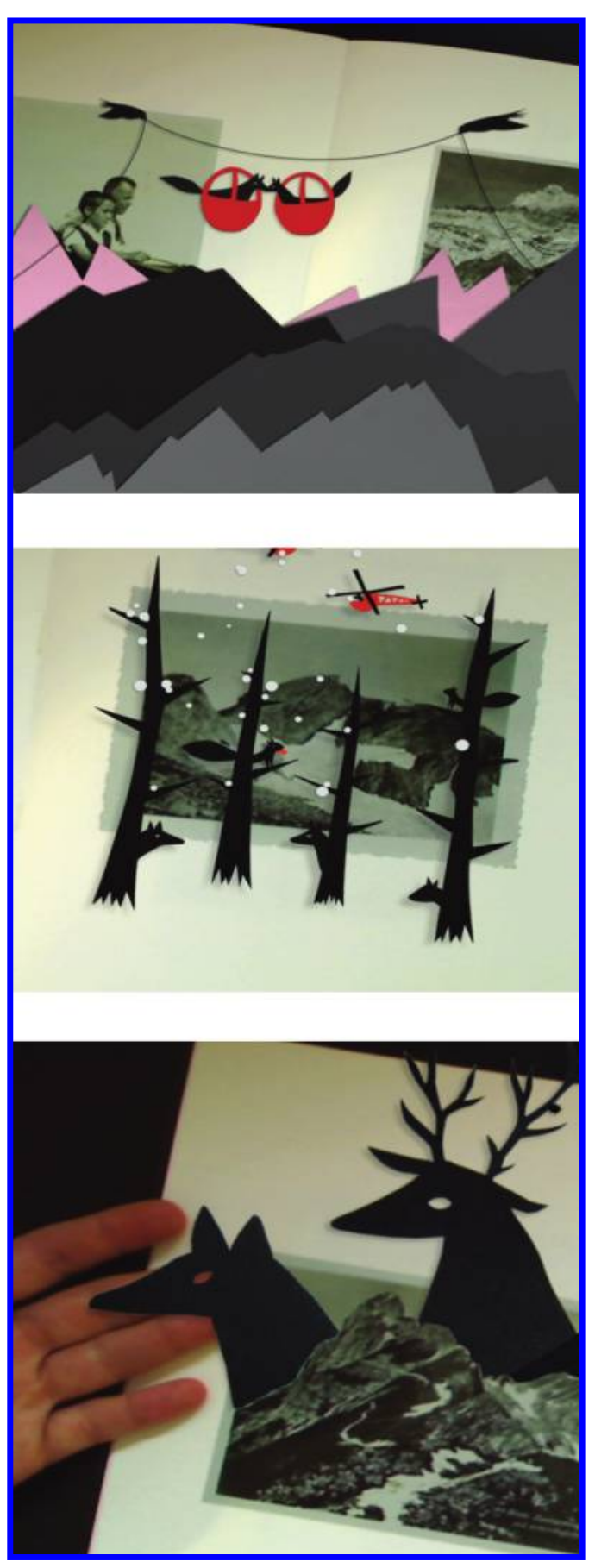

Figure 2. Examples of scenes revealed on the computer screen. The foxes, the deer, and the mountains are added and animated by the computer over the real book. They are designed to look like traditional collages, to blur the border between traditional paper-based media and the virtual world. $\odot 2008$ Camille Scherrer. and paper media have their own role, and both benefit from each other. This contrasts, for example, with a recent commercially published atlas, Atlantica. It features augmented content that seems to simply overlap with the paper book, instead of cooperating with it [5].

When turning the virtual pages of Masaki Fujihata's book with a real pen, the reader is also driven by familiar objects [6]. Fujihata used simple and natural interactions in his virtual book, which we consider as both the source and the strength of this kind of artwork. Our work goes farther and uses a real book as the main driver of the installation. The resulting combination of books and computers creates a new form of interaction in which real and virtual elements have a clearly defined role and visual identity. The interaction principles of many existing augmented-reality applications lack such a clear role assigned to real and virtual components, making some of them less intuitive [7].

Our installation stands out with its particularly convincing merging of paper and computer media (Figure 2). This spectacular questioning of the notion of reality, this inroad into the visual codes of the real and virtual worlds, leads the eye to another interpretation of the book and its representation. The reader/viewer sees objects, their dimensions and iconography, in a different light. Does the process conceal other mysteries, other universes? One does not put this book down like an ordinary read.

\section{Description}

The artistic content of the installation was designed by this paper's first author. Having grown up in the Swiss mountains, surrounded by fir trees, foxes, and squirrels, she wanted to bring this gentle, soothing world together with advanced technology to create new feelings and new meanings. 


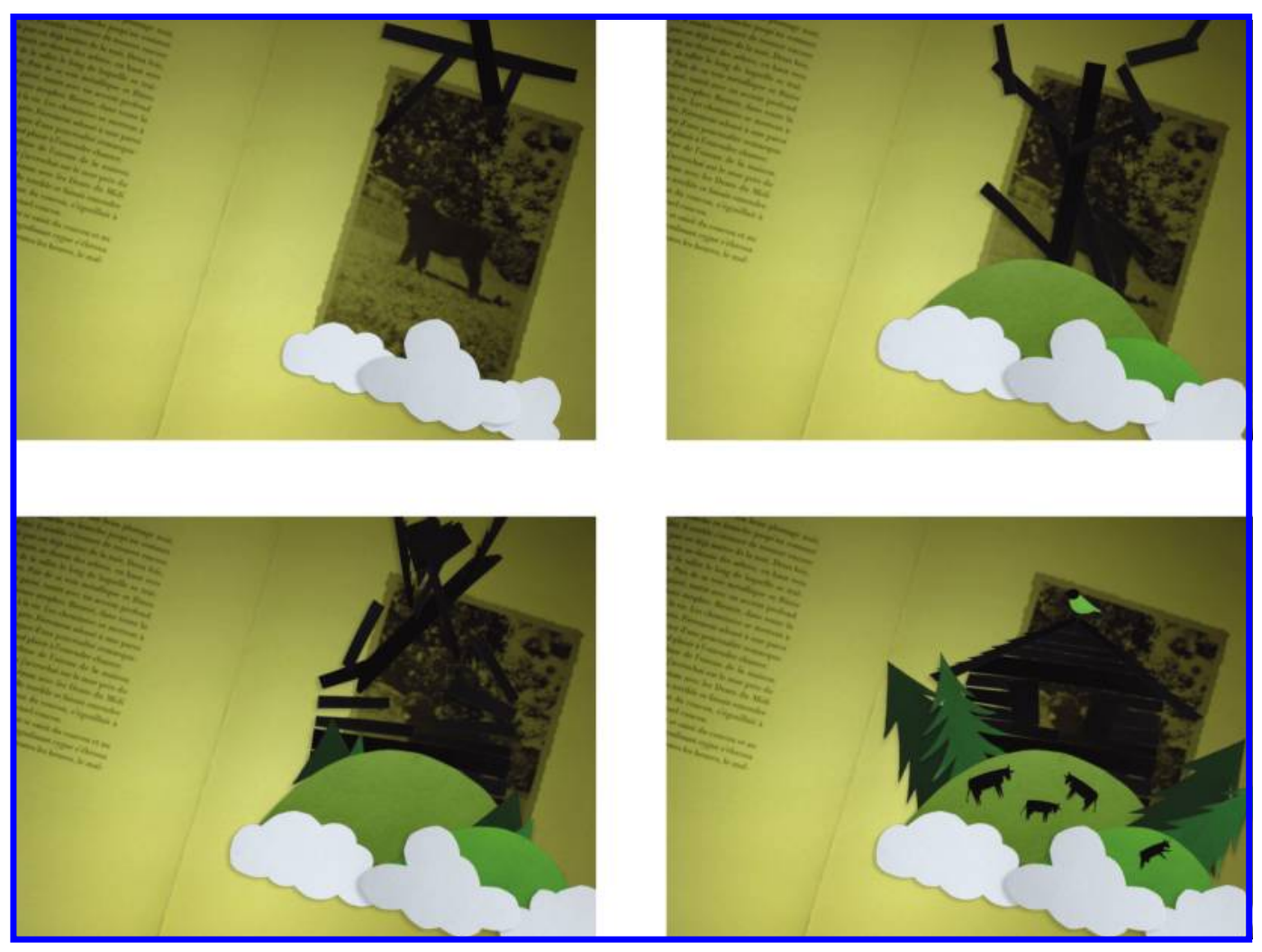

Figure 3. Successive snapshots of an animation. Falling wood planks build up a chalet, and the window opens to reveal the picture behind. ๑ 2008 Camille Scherrer.

The core of our project is to generate interaction between two originally conflicting worlds in order to create a new source of creativity. Between paper and screen, we thought for awhile that the second medium would be the former's downfall, and we describe in this section how our work offers an original way to reconcile or confront both media. We first explain the concept on which our work is based, then describe the design and content we chose to illustrate it. We finally turn to the more technical aspects, to show how the technological part is integrated seamlessly into ordinary objects.

\section{Concept}

Our work offers an original way to reconcile or confront paper and screen, by letting each benefit from the other's intrinsic qualities. Our approach makes the real book-this age-old medium for transferring knowledge — cross the path of the "power book," leading to interaction in a natural, albeit unprecedented, language, as an extension of our reality. The user never feels overwhelmed by technology or bound to a technological constraint.

A book is essentially an ordinary object. As this is the first element of the setup that draws the viewer's attention, the latter is placed in a position of trust and availability. The same applies to the desk lamp, whose familiar shape puts the reader/viewer at ease. The installation therefore stands out from previous setups with obvious technological devices such as cameras, goggles or visual tags [6]. These elements tend not only to discourage the viewer, but also to erect barriers to the human eye between the visible and invisible worlds and to impose markers which kill any questioning by making it obvious where virtual material should be expected. In our case, technology stays discreet by working directly with the normal book content. With the additional hiding 


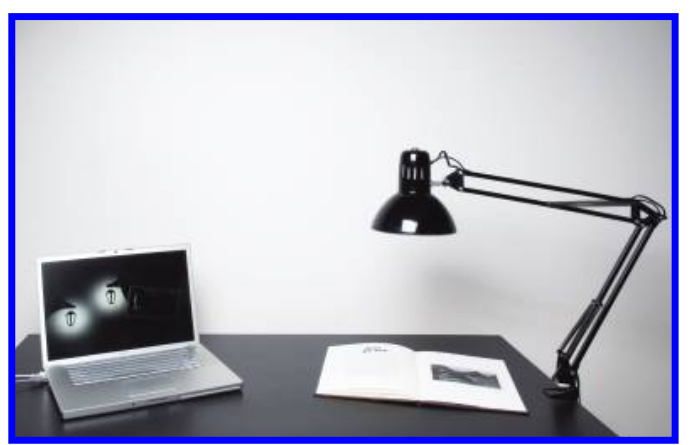

Figure 4. Our setup. The lamp acts as a normal lamp, but also hides a webcam. A standard computer processes the images captured by the webcam and adds the virtual elements. The created images are displayed on the computer screen. ๑) 2008 Daniela Droz.

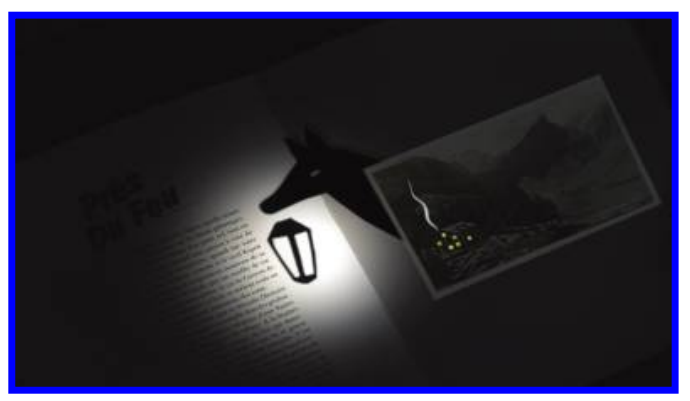

Figure 5. Another example of a revealed scene. The paper and the text are real, and the darkness effect, the fox, and the light are created by the computer. @ 2008 Camille Scherrer. of the camera in the lamp, our setup makes it possible to forget the usual technological constraints and provide a look of authenticity, which is one of the primary drives of this work of art.

Beyond the initial attraction for the reader, the subtle integration of technology into ordinary objects serves to increase the magical aura of the setup. Freed of keys and cursors, the technology becomes more human, as though "swallowed" by paper, and art can appropriate new spaces and invite the viewer to raise questions about objects, information, and technology.

\section{Design \& Content}

The book and animations are designed to produce an immediate emotional impact on the viewer, so as to distract people from any preconceptions about virtual worlds (Figure 3). Through the chosen subjects, shapes, and textures, the artwork plays on the collective unconscious. The visual language stages a sweet nostalgia that brings you back to childhood, to the memories of yesteryear, turning the experience into an epic in direct contact with popular tradition. We have

drawn the iconography from a family album with photographs from the years I9IO to I930 taken at the heart of the Swiss mountains. These images are displayed in a beautifully staged layout that resurrects many references from that era, though one does feel the onset of a time-shift without immediately realizing it. The animations are thus truly startling, as they surge from the universe which readers thought they knew, whereas it eludes them in actual fact. To succeed in surprising and troubling the reader, the animations mix with reality without looking like pasted artifacts. They provide a specific graphic language to complement and blend in with that of the book. The printed images are not a mere background: they seem to contain or even conceal something. Neither the book nor the images are supposed to eclipse each other. They are both essential elements in the setup and equally valuable. Combining them serves to play with the reader/viewer, who sees the frontiers of the familiar universe gradually slip away as a fantasy world emerges.

\section{Setup Description}

As depicted in Figure 4, a lamp, a book, and a computer screen lying on a table make up the visible part of the setup. Other components are hidden. A standard computer runs our software, and the lamp includes an ordinary webcam. So as to strengthen the illusion, the lamp also performs its usual lighting function thanks to a diode system designed to avoid any interference with the camera, such as overheating. Finally, the table is also part of the setup in that it enhances contrasts to provide a consistent stage. The book obeys the overall concept in that in does not include any digital technological element. It could be taken from your grandparents' library. 


\section{Augmented-Reality Technology}

The process we followed to prepare our installation started by designing the book. Because our technology does not require particular marks, we could concentrate on its actual content. The next step was to take digital pictures of each page to be augmented. In the following discussion, we will refer to these pictures as "model images." We then created the virtual animations on top of these model images as animated layers.

During a training phase, our software quickly learns to detect and register the pages in new views based on the model images. It relies on an algorithm based on image-feature matching [8], our implementation of which is available to download [9]. Our software first automatically selects a few hundred characteristic points in each model image and trains a classifier to recognize these points based on their local appearances.

At run time, our software automatically extracts characteristic points in the captured images and matches them in real time against those extracted in the model images using the classifier it built during the training phase. From the matches between the model image and the captured images, it computes the geometric transformation between the two images. Applying this transformation to the semi-transparent animated layer drawn on the corresponding model image yields the augmentation with the appropriate point of view (Figure 5). Because our technology is automatic, easy to use, and robust to illumination changes, we can reliably let the public enjoy our installation without assistance.

\section{Conclusion}

The first stages of this work, initiated as part of a diploma project [Io], have been widely recognized, in particular with the prestigious Pierre Bergé award for the best European design diploma. This success stems from an innovative artistic exploration into the notions of augmented reality, involving both technological advancements and development of visual communication. More generally speaking, the project tantalizes our perception of the virtual and real worlds by taking us through an unprecedented experience. We feel that Souvenirs du monde des montagnes can offer another view of virtuality to the public. The topic we used and the approach we adopted have already convinced unexpected audiences from five to 95 years old: when Pierre Bergé, an 80-year-old art connoisseur, looked at our artwork, he immediately felt comfortable and knew how to play with it. Our group aims at developing new links between design and engineering, and we consider this work an example of renewed dialogue between the two disciplines. Our approach to augmented reality, which allows paper and computer to meet concretely and in harmony, solves the inherent competition between the real and virtual worlds. 


\section{References}

I. M. Bataille, $A B C_{3} d$ (Paris: Albin Michel Jeunesse, 2008).

2. M. Billinghurst, H. Kato, I. Poupyrev, "The MagicBook-Moving Seamlessly Between Reality and Virtuality," IEEE Computer Graphics and Applications, Vol. 2I, No. 3, 6-8 (200I).

3. R. Grasset, A. Duenser, M. Billinghurst, "The Design of a Mixed-Reality Book: Is It Still a Real Book?," Proceedings of the International Symposium on Augmented Reality, 99-102 (2008).

4. T. Saso, K. Iguchi, and M. Inakage, "Little red: Storytelling in mixed reality," Proceedings of ACM SIGGRAPH Sketches \& Applications (2003).

5. Atlantica Der grosse Weltatlas (Munich, Germany: Wissenmedia GmbH, 2008).

6. M. Fujihata, "Beyond Pages," Leonardo, Vol. 35, No. 5, 545 (2002).

7. H. Kato, M. Billinghurst, I. Poupyrev, K. Imamoto, K. Tachibana, "Virtual Object Manipulation on a Table-Top AR Environment," Proceedings of the International Symposium on Augmented Reality, III-II9 (2000).

8. M. Ozuysal, P. Fua, and V. Lepetit, "Fast Keypoint Recognition in Ten Lines of Code," Proceedings of the Conference on Computer Vision and Pattern Recognition (Minneapolis, MN, 2007).

9. http://cvlab.epfl.ch/software/ferns.

Io. Media \& Interaction Design, ECAL/University of Art and Design, Lausanne, Switzerland. 